\title{
PSFC/JA-06-32
}

\section{Effects of finite poloidal gyroradius, shaping, and collisions on the zonal flow residual}

\author{
${ }^{1}$ Yong Xiao, ${ }^{2}$ Peter J. Catto, and ${ }^{3}$ William D. Dorland
}

\author{
November 2006 \\ ${ }^{1}$ University of California Irvine, Irvine, CA 92697, USA \\ ${ }^{2}$ MIT Plasma Science and Fusion Center, 167 Albany Street, Cambridge, \\ MA 02139, USA \\ ${ }^{3}$ Institute for Plasma Research, University of Maryland, College Park, \\ Maryland 20742, USA
}

This work was supported by the U.S. Department of Energy, Grant No. DE-FG02-91ER-54109 Submitted for publication in the Physics of Plasma 


\title{
Effects of finite poloidal gyroradius, shaping, and collisions on the zonal flow residual
}

\author{
Yong Xiao and Peter J. Catto \\ MIT Plasma Science and Fusion Center, Cambridge, MA 02139, U.S.A. \\ William D. Dorland \\ Institute for Plasma Research, University of Maryland, \\ College Park, Maryland 20742, USA
}

(Dated: 10/17/2006)

\begin{abstract}
Zonal flow helps reduce the turbulent transport level in tokamaks. Rosenbluth and Hinton have shown that zonal flow damps to to a non-vanishing residual level in collisionless and collisional banana regime plasmas Recent zonal flow advances are summarized including the evaluation of the effects on this zonal flow residual of plasma cross section shaping, shorter wavelengths including those less than an electron gyroradius, and aritrary collisionality relative to the zonal low frequency. In addition to giving a brief summary of these new developments, the analytic results are compared with GS2 numerical simulations to demonstarte their value as benchmarks for turbulence codes.
\end{abstract}




\section{INTRODUCTION}

Recent discoveries in plasma turbulence show that zonal flow is an important mechanism for suppressing ion temperature gradient (ITG) [1][2][3] and trapped electron mode (TEM) [4][5] turbulence. As a result, it is important to understand the damping mechanisms that act on zonal flow. The original Rosenbluth-Hinton (R-H) study showed that zonal flow is modified by the collisionless neoclassical polarization, with a significant residual flow surviving due to the smallness of this polarization [6]. Later, Hinton-Rosenbluth (H-R) found that in the large radial wavelength limit collisional effects significantly reduce the residual zonal flow to a level much smaller [7] than the collisionless kinetic theory predicts [6]. Both of these analytical studies are based on a large aspect ratio circular flux surface tokamak model.

These classical studies on the linear damping of zonal flow have been extended in many aspects recently. First of all, in the large radial wavelength limit, the original R-H collisionless residual zonal flow calculation [6] has been extended to more realistic flux surface shapes that allow elongation, triangularity and Shafranov shift to be retained in the equilibrium model [8]. Secondly, the original R-H collisionless calculation has been extended to cover arbitrary radial wavelength zonal flow [9], including in particular shorter wavelength effects for ITG and TEM modes and even the short wavelengths associated with electron temperature gradient (ETG) turbulence. Finally, a new method has been developed to study collisional zonal flow damping [10]. This new analytical approach is valid for arbitrary collisionalities and long wavelengths, and therefore a useful extension of the original H-R collisional work $[11][12]$.

These new developments in the $\mathrm{R}-\mathrm{H}$ and $\mathrm{H}-\mathrm{R}$ zonal flow studies provide not only new insights on the physics of residual zonal flow, but also new opportunities to crosscheck numerical simulations. Some of these checks for the well-known continuum turbulence code GS2 are presented here. The GS2 code had previously successfully benchmarked the wellknow R-H collisionless residual zonal flow calculation [13]. This code also discovered the increase of residual zonal flow for short radial wavelength zonal flow [14] driven by ETG, ITG and TEM turbulence. Moreover, a recent GS2 study on shaping effects [15] has given results similar to our analytical theory. Therefore, it is desirable to make a more thorough and careful comparison between the new analytical developments [8][9][10] and the numerical 
simulations using the GS2 code under common circumstances and parameters.

This paper is organized as follows. Sections II and III review the linear gyrokinetics of zonal flow and the recent extensions of the $\mathrm{R}-\mathrm{H}$ and $\mathrm{H}-\mathrm{R}$ zonal flow models. In Sec. IV, we briefly summarize the new analytical results for plasma shaping effects and compare them to the GS2 numerical simulations. Section V briefly reviews the recent analytical calculation of collisionless residual zonal flow for the arbitrary radial wavelength and compares it to the GS2 simulation result. In Sec. VI, we compare the collisional damping of zonal flow from the GS2 code to the recent analytical result. Finally, Sec. VII provides a brief discussion highlightening the newly discovered results including the comparisons discussed in the preceding sections.

\section{LINEAR GYROKINETICS DRIVEN BY ZONAL FLOW}

The linearized gyrokinetic equation can be employed to study the linear response of the plasma to an axisymmetric zonal flow potential caused by turbulence[6]. The distribution function is assumed to be composed of two parts: the unperturbed and the perturbed part. The unperturbed part is assumed to be a radially slowly varying Maxwellian $F_{0}$. The

perturbed part is driven by the zonal flow potential, and has the form $f=-\frac{e \phi}{T} F_{0}+g$, where the adiabatic response has been separated out for convenience. Here we assume all the perturbed quantities take an eikonal form $\phi=\sum_{k} \phi_{k} e^{i S}$ with the eikonal $S=S(\psi)$ and the radial wave vector $\mathbf{k}_{\perp}=\nabla S$. Then in the Fourier space, the guiding center distribution $g_{k}$ satisfies the following gyrokinetic equation, [6][16][17]

$$
\frac{\partial g_{k}}{\partial t}+\left(v_{\|} \mathbf{b} \cdot \nabla+i \omega_{D}\right) g_{k}-C\left\{g_{k}\right\}=-\frac{e}{T_{i}} F_{0} J_{0} \frac{\partial \phi_{k}}{\partial t}
$$

where $C$ is the gyroaveraged collision operator, $J_{0}$ is the zeroth order Bessel function, $J_{0}=$ $J_{0}\left(\frac{k_{\perp} v_{\perp}}{\Omega}\right)$, and $\omega_{D}=\mathbf{k}_{\perp} \cdot \mathbf{v}_{d}=v_{\Perp} \mathbf{b} \cdot \nabla Q$ with $Q=I S^{\prime} v_{\Perp} / \Omega$ coming from the magnetic drift $\mathbf{v}_{d}=\frac{\mathbf{b}}{\Omega} \times\left(\mu \nabla B+v_{\|}^{2} \mathbf{b} \cdot \nabla \mathbf{b}\right)$ since $\mathbf{v}_{d} \cdot \nabla \psi=v_{\|} \mathbf{b} \cdot \nabla\left(\frac{I v_{\|}}{\Omega}\right)$. Notice $Q \sim k_{\perp} \rho_{p}$, where $\rho_{p}$ is the poloidal gyroradius. The independent velocity variables used in the preceding equation are kinetic energy $E=v^{2} / 2$ and magnetic moment $\mu=v_{\perp}^{2} / 2 B$. For simplicity, hereinafter we assume hydrogenic ions in the plasma.

Since the zonal flow frequency $\omega$ considered here is far below the transit frequency of thermal particles $\omega_{t}=v_{T} / q R_{0}$, the equation can be solved perturbatively by expanding in 
$\omega / \omega_{t} \ll 1[6]$ by letting $g_{k}=g_{k}^{0}+g_{k}^{1}+\ldots$. The leading order equation in this expansion gives

$$
v_{\|} \mathbf{b} \cdot \nabla g_{k}^{0}+i v_{\|} g_{k}^{0} \mathbf{b} \cdot \nabla Q=0,
$$

whose solution has the form $g_{k}^{0}=h_{k} e^{-i Q}$ with $\mathbf{b} \cdot \nabla h_{k}=0$. Then next order equation gives

$$
v_{\|} \mathbf{b} \cdot \nabla g_{k}^{1}+i v_{\|} g_{k}^{1} \mathbf{b} \cdot \nabla Q g_{k}^{1}=-\frac{\partial g_{k}^{0}}{\partial t}+C\left\{g_{k}^{0}\right\}_{\varphi}-\frac{e}{T_{i}} F_{0} J_{0} \frac{\partial \phi_{k}}{\partial t} .
$$

The transit average of the product of this equation and $e^{i Q}$ gives

$$
\frac{\partial h_{k}}{\partial t}-\overline{e^{i Q} C\left\{h_{k} e^{-i Q}\right\}}=-\frac{e}{T} F_{0} \overline{J_{0} e^{i Q}} \frac{\partial \phi_{k}}{\partial t},
$$

where the transit average is defined as, $\bar{A}=\oint d \tau A / \oint d \tau$, with $d \tau=d \theta /\left(v_{\|} \mathbf{b} \cdot \nabla \theta\right)$. For trapped particles, this average is over a full bounce; while for passing particles, it is over one complete poloidal circuit. Specifically, for a large aspect ratio circular cross section tokamak, $d \tau \cong q R_{0} d \theta / v_{\|}$, where $q$ is the safety factor. In this case the transit average becomes

$$
\bar{A}=\frac{\oint \frac{d \theta}{v_{\|}} A}{\oint \frac{d \theta}{v_{\|}}} .
$$

When calculating the perturbed particle density in a flux surface, we utilize

$$
\tilde{n}_{k}=\left\langle\int d^{3} v J_{0} h_{k} e^{-i Q}\right\rangle-\frac{e \phi_{k}}{T} n_{0},
$$

where \langle\rangle represents the flux surface average $\langle A\rangle=\oint \frac{d l}{B} A / \oint \frac{d l}{B}$.

If the time scale of interest is much shorter than a typical collision time, the plasma can treated as collisionless; otherwise collisions must be retained. For the collisionless case, the solution to the transit average kinetic equation, Eq. (4), is straightforward

$$
h_{k}=\frac{e \phi_{k}}{T} F_{0} \overline{J_{0} e^{i Q}} .
$$

Hence, the perturbed particle density in Eq.(6) can be expressed as

$$
\tilde{n}_{k}=\frac{e \phi_{k}}{T} n_{0}\left(\frac{1}{n_{0}}\left\langle\int d^{3} v J_{0} e^{-i Q} \overline{J_{0} e^{i Q}} F_{0}\right\rangle-1\right),
$$

where the classical gyro-motion effect (finite Larmor radius) on polarization is retained in $J_{0}$, and the effect of magnetic drift (finite poloidal gyroradius) is retained in $e^{i Q}$.

For the collisional case, the transit average kinetic equation (4) can only be solved for large radial wavelength zonal flow where $k_{\perp} \rho_{p i} \ll 1$. The ITG and TEM mode driven zonal flows fall into this category. Expanding Eq. (4) and (6) to order $Q^{2}$ [7][10], we find

$$
\tilde{n}_{k}=-\frac{e \phi_{k}}{T} n_{0}\left[\left\langle k_{\perp}^{2} \rho^{2}\right\rangle+\frac{1}{n_{0}}\left\langle\int d^{3} v F_{0}\left(Q^{2}+\frac{i Q T h_{k}^{(1)}}{e \phi_{k} F_{0}}\right)\right\rangle\right] .
$$


where the distribution function $h_{k}^{(1)}$ satisfies

$$
\frac{\partial h_{k}^{(1)}}{\partial t}-\overline{C\left\{h_{k}^{(1)}\right\}}=i \bar{Q} \frac{e}{T} F_{0} \frac{\partial \phi_{k}}{\partial t} .
$$

We define the gyroradius as $\rho=\sqrt{T / m} / \Omega$ with the gyro frequency $\Omega=e B / m c$.

Next we will review the Rosenbluth-Hinton zonal flow physics and its relationship to the current linear density calculation.

\section{GENERALIZED ROSENBLUTH-HINTON ZONAL FLOW PHYSICS}

Quasineutrality of the plasma requires that the linear perturbed charge density be compensated by the nonlinear turbulent charge source, i. e., $e \tilde{n}_{k}^{(i)}-e \tilde{n}_{k}^{(e)}=-\rho_{k}^{N L}$. In the Rosenbluth-Hinton zonal flow model, turbulence produces a constant initial charge source within a time that is much shorter than one transit time but much larger than one gyroperiod. Therefore, drift and collisional effects can be ignored $(Q=0=C)$ on such short time scales and the initial zonal flow potential is given by

$$
\begin{aligned}
& {\left[\tau\left(\frac{1}{n_{0}}\left\langle\int d^{3} v{\overline{J_{0 i}}}^{2} F_{0 i}\right\rangle-1\right)+\left(\frac{1}{n_{0}}\left\langle\int d^{3} v{\overline{J_{0 e}}}^{2} F_{0 e}\right\rangle-1\right)\right] \frac{n_{0} e^{2}}{T_{e}} \phi_{k}(t=0)} \\
& =\rho_{k}^{N L}(0),
\end{aligned}
$$

according to Eq.(8) and quasineutrality, where $\tau=T_{e} / T_{i}$. For the long wavelength ITG and/or TEM zonal flow cases $k_{\perp} \rho_{p i} \ll 1$, this equation simplifies considerably to become

$$
\frac{n_{0} e^{2}}{T_{i}} \phi_{k}(t=0)\left\langle k_{\perp}^{2} \rho_{i}^{2}\right\rangle=\rho_{k}^{N L}(0) .
$$

After several transit periods, drift effects become important. In the collisionless limit, the long time zonal flow is then given by

$$
\begin{aligned}
& {\left[\tau\left(\frac{1}{n_{0}}\left\langle\int d^{3} v J_{0 i} e^{-i Q_{i}} \overline{J_{0 i} e^{i Q_{i}}} F_{0 i}\right\rangle-1\right)+\left(\frac{1}{n_{0}}\left\langle\int d^{3} v J_{0 e} e^{-i Q_{e}} \overline{J_{0 e} e^{i Q_{e}}} F_{0 e}\right\rangle-1\right)\right] \frac{n_{0} e^{2}}{T_{e}} \phi_{k}(t=\infty)} \\
& =\rho_{k}^{N L}(0) .
\end{aligned}
$$

Therefore, the zonal flow residual, that is customarily $\phi_{k}(t=\infty) / \phi_{k}(t=0)$, has the form

$$
\begin{aligned}
& \frac{\phi_{k}(t=\infty)}{\phi_{k}(t=0)} \\
& =\frac{\tau\left(\frac{1}{n_{0}}\left\langle\int d^{3} v{\overline{J_{0 i}}}^{2} F_{0 i}\right\rangle-1\right)+\left(\frac{1}{n_{0}}\left\langle\int d^{3} v{\overline{J_{0 e}}}^{2} F_{0 e}\right\rangle-1\right)}{\tau\left(\frac{1}{n_{0}}\left\langle\int d^{3} v J_{0 i} e^{-i Q_{i}} \overline{J_{0 i} e^{i Q_{i}}} F_{0 i}\right\rangle-1\right)+\left(\frac{1}{n_{0}}\left\langle\int d^{3} v J_{0 e} e^{-i Q_{e}} \overline{J_{0 e} e^{i Q_{e}}} F_{0 e}\right\rangle-1\right)}
\end{aligned}
$$


In the long wavelength limit $k_{\perp} \rho_{p i} \ll 1$, the collisionless zonal flow residual reduces to

$$
\frac{\phi_{k}(t=\infty)}{\phi_{k}(t=0)}=\frac{\left\langle k_{\perp}^{2} \rho_{i}^{2}\right\rangle}{\left\langle k_{\perp}^{2} \rho_{i}^{2}\right\rangle+\frac{1}{n_{0}}\left\langle\int d^{3} v F_{0 i}\left(Q_{i}^{2}-{\overline{Q_{i}}}^{2}\right)\right\rangle} .
$$

When the time scales of interest are comparable to a typical collision time, the collisional effects becomes significant. At present, the collisional case is only tractable for the large wavelength zonal flow. In this case, the bounce average drift kinetic equation, Eq. (10), must be solved to evaluate the linearized particle density in Eq.(9). It is generally more convenient to solve Eq. (10) in the frequency domain,

$$
h_{k}^{(1)}(p)-\frac{1}{p} \overline{C\left\{h_{k}^{(1)}(p)\right\}}=i \bar{Q} \frac{e}{T} F_{0} \phi_{k}(p),
$$

which comes from the Laplace transform of Eq. (10). The Laplace transforms of $\phi_{k}$ and $h_{k}^{(1)}$ are defined by $\phi_{k}(p)=\int_{0}^{\infty} d t e^{-p t} \phi_{k}(t)$ and $h_{k}^{(1)}(p)=\int_{0}^{\infty} d t e^{-p t} h_{k}^{(1)}(t)$, where $p$ is the frequency variable. In the frequency domain, the quasineutrality condition becomes

$$
\frac{n_{0} e^{2}}{T_{i}} \phi_{k}(p)\left[\left\langle k_{\perp}^{2} \rho_{i}^{2}\right\rangle+\frac{1}{n_{0}}\left\langle\int d^{3} v F_{0 i}\left(Q_{i}^{2}+\frac{i Q_{i} T_{i} h_{k}^{(1)}(p)}{e \phi_{k} F_{0}}\right)\right\rangle\right]=\frac{\rho_{k}^{N L}(0)}{p},
$$

where the distribution $h_{k}^{(1)}(p)$ satisfies Eq. (17) for ions. The electron perturbed charge density is normally ignored because it is a mass ratio smaller than the ion part for the ITG and TEM zonal flow. This equation together with Eq. (13) give the frequency response of the zonal flow to be

$$
\frac{\phi_{k}(p)}{\phi_{k}(t=0)}=\frac{\left\langle k_{\perp}^{2} \rho_{i}^{2}\right\rangle / p}{\left\langle k_{\perp}^{2} \rho_{i}^{2}\right\rangle+\frac{1}{n_{0}}\left\langle\int d^{3} v F_{0 i}\left(Q_{i}^{2}+\frac{i Q_{i} T_{i} h_{k}^{(1)}(p)}{e \phi_{k} F_{0}}\right)\right\rangle} .
$$

The inverse Laplace transform of this equation gives the time evolution of zonal flow

$$
\frac{\phi_{k}(t)}{\phi_{k}(t=0)}=\frac{1}{2 \pi i} \int d p e^{p t} \frac{\left\langle k_{\perp}^{2} \rho_{i}^{2}\right\rangle / p}{\left\langle k_{\perp}^{2} \rho_{i}^{2}\right\rangle+\frac{1}{n_{0}}\left\langle\int d^{3} v F_{0 i}\left(Q_{i}^{2}+\frac{i Q_{i} T_{i} h_{k}^{(1)}(p)}{e \phi_{k} F_{0}}\right)\right\rangle} .
$$

Therefore, the long time behavior of zonal flow or the zonal flow residual is determined by the zero frequency response of Eq.(19), and the damping rate of the zonal flow is determined by the zeroes of the term $\left\langle k_{\perp}^{2} \rho_{i}^{2}\right\rangle+\frac{1}{n_{0}}\left\langle\int d^{3} v F_{0 i}\left(Q_{i}^{2}+\frac{i Q_{i} T_{i} h_{k}^{(1)}(p)}{e \phi_{k} F_{0}}\right)\right\rangle$.

The following sections will briefly discuss three different recent developments that extend the $\mathrm{R}-\mathrm{H}$ and $\mathrm{H}-\mathrm{R}$ zonal flow calculations, and briefly present comparisons to the corresponding GS2 simulations. 


\section{PLASMA SHAPING EFFECTS ON ZONAL FLOW RESIDUAL}

The shaping factors of magnetic flux surfaces, like elongation $\kappa$ and triangularity $\delta$, are important ingredients in suppressing turbulent transport in tokamaks [18]. Recent numerical [15] and analytical [8] studies show that shaping also affects the collisionless residual zonal flow level. Although these two approaches are based on different equilibrium models, they show similar dependences on plasma shaping. Here we provide a more careful comparison between the analytical formula and GS2 numerical simulation.

The analytical approach applies a global equilibrium[19], whose flux surface in the large aspect ratio limit can be simplified as [8]

$$
\begin{aligned}
& R=R_{0}\left(1+\varepsilon \cos \theta-\Delta \cos ^{2} \theta-\frac{\kappa^{2}}{4 E^{2}} \varepsilon^{2} \sin ^{2} \theta\right), \\
& Z=R_{0} \kappa \varepsilon \sin \theta
\end{aligned}
$$

while keeping the important shapings, such as elongation $\kappa$, triangularity $\delta$, and Shafranov shift $\Delta$ in the model. For a large aspect ratio tokamak, an $\varepsilon$ expansion can be applied to calculate the zonal flow residual in Eq. (16) to obtain

$$
\frac{\phi_{k}(t=\infty)}{\phi_{k}(t=0)}=\frac{1}{1+S q^{2} / \sqrt{\varepsilon}}
$$

with the shaping function $S$ given by

$$
\begin{aligned}
S & =\frac{1}{1+\kappa^{2}}\left(3.27+\sqrt{\varepsilon}+0.722 \varepsilon-1.443 \delta-2.945 \frac{\Delta}{\varepsilon}\right. \\
& \left.+\frac{0.692 \kappa^{2}-0.722}{q^{2}} \varepsilon\right) .
\end{aligned}
$$

This analytical result can be compared to GS2 simulations, as shown in Figs. 1 and 2. In Fig. 1, the elongation dependence of the zonal flow residual are compared to GS2 simulation, and showing very good agreement. In Fig. $2, \kappa=1.8$, and the triangularity dependence of the zonal flow residual is compared to GS2 simulation. The slope of these two curves are the same, telling us that the coefficient of the dependence of $\delta$ in the shaping function are the same. The small difference is due to the differing $\varepsilon$ dependence of the global analytical and local numerical equilibrium models.

We see that the leading order effect of shaping is due to elongation, which comes from the increase of the poloidal field $B_{p}$ with elongation $\kappa$ when keeping the safety factor $q$ fixed. The triangularity $\delta$ effect is due to the change of the trapped-passing boundary location and therefore the change in the ratio between the trapped and passing particles. 


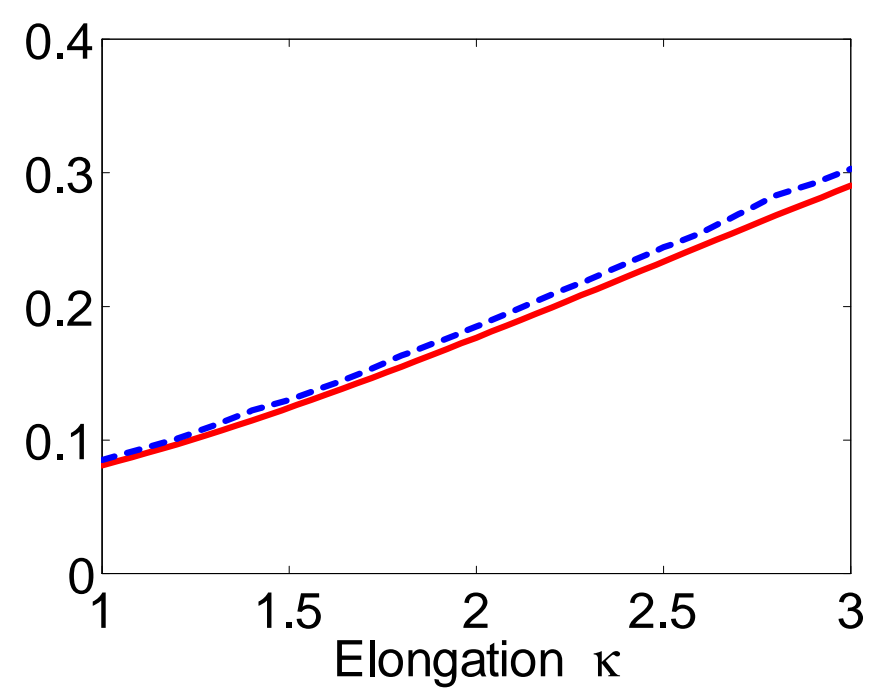

FIG. 1: Zonal flow residual dependence on elongation $\kappa$, for $q=1.4, \delta=0, \Delta=0$. The solid line is the analytical result. The dashed line is the GS2 simulation result.

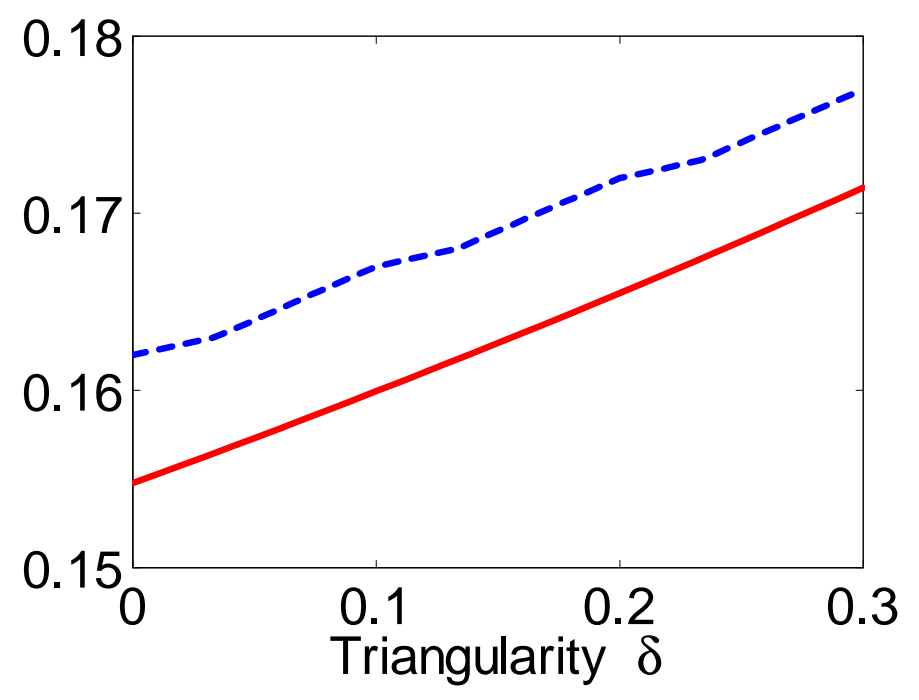

FIG. 2: Zonal flow residual dependence on elongation $\delta$, for $q=1.4, \kappa=1.8, \Delta=0$. The solid line is the analytical result. The dashed line is the GS2 simulation result.

\section{SHORT WAVELENGTH EFFECTS ON ZONAL FLOW RESIDUAL}

The pioneering calculation of R-H [6] focused on ITG mode driven zonal flow in the large wavelength limit satisfying $k_{\perp} \rho_{p i} \ll 1$. However, both experiments [20] and simulations [21] 
show that these zonal flows can have radial wavelength comparable to the ion poloidal gyroradius. Moreover, sources of anomalous transport such as the trapped electron mode (TEM) [4][5] and electron temperature gradient (ETG) mode [11][12] can also drive zonal flows at shorter radial wavelengths than the ion gyroradius. Indeed, for ETG turbulence wavelengths as shorter than the electron gyroradius must be considered. Such short wavelength zonal flows weren't considered by the original R-H calculation. Here we briefly summarize a recent study of the short wavelength effects collisionless zonal flow damping [9].

Before doing so we recall that when the radial wavelength is much larger than the ion poloidal gyroradius, $k_{\perp} \rho_{p i} \ll 1$, Eq.(16) leads to the R-H value of the zonal flow residual

$$
\begin{aligned}
\frac{\phi_{k}(t=\infty)}{\phi_{k}(t=0)} & =\frac{1}{1+\left\langle\int d^{3} v F_{0 i}\left(Q_{i}^{2}-{\overline{Q_{i}}}^{2}\right)\right\rangle / n_{0}\left\langle k_{\perp}^{2} \rho_{i}^{2}\right\rangle} \\
& =\frac{1}{1+\frac{q^{2}}{\varepsilon^{2}}\left(1.6 \varepsilon^{3 / 2}+0.5 \varepsilon^{2}+0.36 \varepsilon^{5 / 2}\right)},
\end{aligned}
$$

which doesn't depend on the radial wavenumber $k_{\perp}$. The higher order terms retained in the preceding expression make the result more accurate for the finite size $\varepsilon$ values than the original R-H coefficient $1.6 \varepsilon^{3 / 2}[9]$.

When the radial wavelength becomes close to the ion poloidal gyroradius, the finite poloidal gyroradius effect tries to increase the residual zonal flow level,

$$
\frac{\phi_{k}(t=\infty)}{\phi_{k}(t=0)}=\frac{1}{1+\frac{q^{2}}{\varepsilon^{2}}\left[\left(1.6 \varepsilon^{3 / 2}+0.5 \varepsilon^{2}+0.36 \varepsilon^{5 / 2}\right)-2.44 \varepsilon^{5 / 2} k_{\perp}^{2} \rho_{p i}^{2}\right]} .
$$

This result is valid for $k_{\perp} \rho_{i} \ll 1$ and $k_{\perp} \rho_{p i}$ approaching unity [9].

As the radial wavelength gets even shorter, Eq. (15) needs to be numerically evaluated to obtain the collisionless zonal flow residual. When the radial wavelengths are small compared to an ion gyroradius, but comparable to or less than a poloidal electron gyroradius, electron polarization becomes important. The zonal flow residual first decreases with $k_{\perp}$ due to electron neoclassical polarization. Then the zonal flow residual recovers and increases due to finite electron poloidal gyroradius effects. Finally, as the radial wavelengths becomes much less than an electron gyroradius, the zonal flow residual ultimately approaches unity.

The preceding behavior is what Jenko et. al. observed in GS2 simulations for ETG modes [14], but for a slightly different driving source function. A careful comparison between the predictions of Eq. (15) and GS2 for the same source functions and parameters has now 


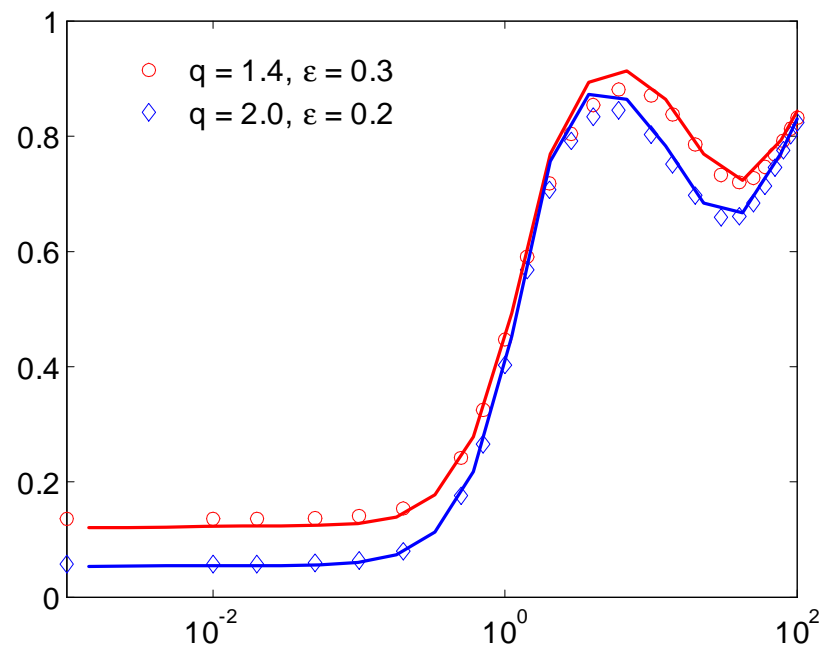

FIG. 3: The zonal flow residual $\phi_{k}(t=\infty) / \phi_{k}(t=0)$ varies with normalized radial wavelength $k_{\perp} \rho_{i}$. The discrete shapes are from GS simulation. The solid lines are based on Eq.(15) and from Ref. [9].

been performed to obtain the plots shown in Fig. 1. The agreement between these two independent calculations is excellent and provides a useful benchmark of the zonal flow residual for arbitrary radial wavelengths.

Based on this figure, we expect ETG turbulence to saturate at a low level if short wavelengths are generated by the parasitic turbulence associated with ETG, ITG, and/or TEM modes. The high level of ETG turbulence sometimes observed in codes may indicate that the parasitic instabilities associated with ETG modes are not as effective in generating zonal flow as those associated with ITG and TEM modes [14][22].

\section{COLLISIONAL ZONAL FLOW DAMPING FOR LARGE WAVELENGTH ZONAL FLOW}

When the time scale of interest is on the order of the ion-ion collisional time $\tau_{i i}$, the collisional damping of zonal flow becomes important. The original H-R calculation considers two asymptotic limits: the short time limit $\varepsilon p \tau_{i i} \gg 1$ and the long time limit $p \tau_{i i} \ll 1$. However, to obtain an improved estimate of the decay rate it is desirable to obtain the frequency response of Eq. (19) for arbitrary values of $p \tau_{i i}$. A new method based on an 


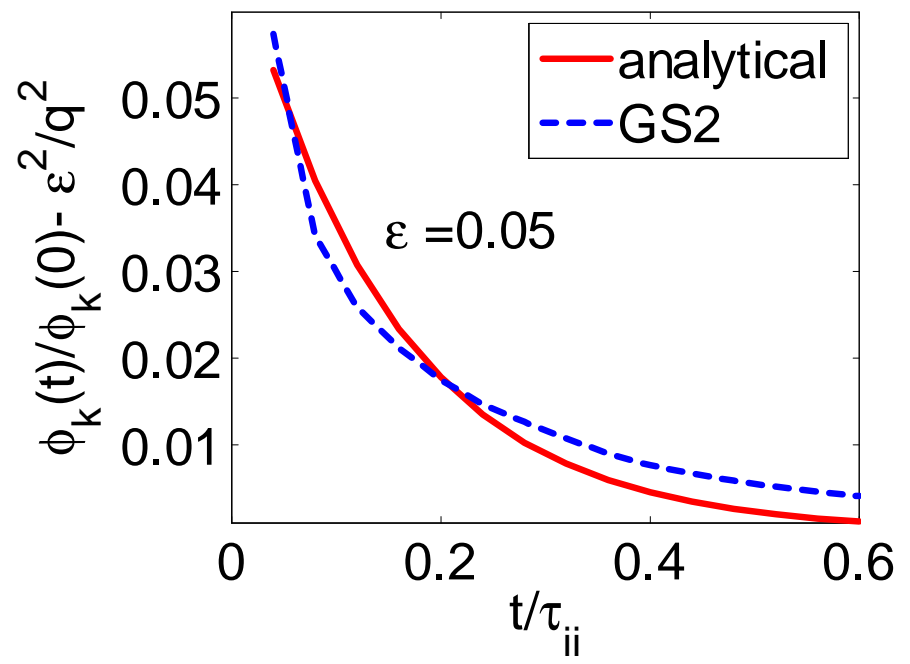

FIG. 4: A comparison of collisional zonal flow damping from both analytical theory and GS2 simulation.

eigenfunction expansion of the pitch angle scattering operator has been developed to solve this problem [10]. It finds that in the large wavelength limit, Eq. (19) can be approximated as

$$
\frac{\phi_{k}(t)}{\phi_{k}(t=0)} \approx \frac{\varepsilon^{2}}{q^{2}}\left[1+\left(\frac{1}{1.6 \varepsilon^{3 / 2}}-1\right) e^{-\frac{\mu_{1} t}{1.6 \varepsilon^{3 / 2} \eta_{1} \tau_{i i}}}\right]
$$

where $\mu_{1}=1+1.46 \sqrt{\varepsilon}$ and $\eta_{1}=1.16 / \sqrt{\varepsilon}+5.38$. This analytical result can now be compared to the GS2 numerical simulation. For small $\varepsilon$, such as $\varepsilon=0.05$, our analytical result captures the main feature of the decay curve from the GS2 simulation. As $\varepsilon$ becomes larger, our analytical result becomes more approximate.

\section{DISCUSSION AND CONCLUSION}

This paper provides a systematic review of the R-H collisionless and H-R collisional zonal flow damping and their recent extensions in three different directions. These three important extensions have been carefully tested by GS2 simulation. In the collisionless regime, the shaping effects, like elongation and triangularity, have been confirmed by GS2 simulation. Elongation, or the increase of poloidal field, is seen to substantially increase the zonal flow residual, and may have important consequences in regulating turbulence. Short wavelength effects on the zonal flow residual are also evaluated analytically and by GS2 simulation 
and found to be in agreement to great accuracy. The increase of zonal flow residual for short wavelengths may indicate there is a strong impact on ETG turbulence provided that instabilities produces the same amount of zonal flow as the ITG and TEM modes. Finally we provide a preliminary study of the collisional damping of zonal flows between the GS2 simulation and the analytical result from our new approach, that by comparing improves on the H-R result. Good consistency is achieved in the large aspect ratio limit.

[1] W. Dorland and G. Hammett, Phys. Fluids B 5, 812 (1993).

[2] Z. Lin, T. Hahm, W. Lee, W. Tang, and R. White, Science 281, 1835 (1998).

[3] J. Candy and R. Waltz., Phys. Rev. Lett. 91, 045001 (2003).

[4] D. Ernst et al., Phys. Plasmas 11, 2637 (2004).

[5] B. Coppi and G. Rewoldt, Phys. Rev. Lett. 33, 1329 (1974).

[6] M. Rosenbluth and F. Hinton, Phys. Rev. Lett. 80, 724 (1998).

[7] F. Hinton and M. Rosenbluth, Plasma Phys. Control. Fusion 41, A653 (1999).

[8] Y. Xiao and P. Catto, Phys. Plasmas 13, 082307 (2006).

[9] Y. Xiao and P. Catto, submitted to Phys. Plasmas (2006).

[10] Y. Xiao, P. Catto, and K. Molvig, submitted to Phys. Plasmas (2006).

[11] W. Dorland, F. Jenko, M. Kotschenruther, and B. Rogers, Phys. Rev. Lett. 85, 5579 (2000).

[12] Y. Lee, J. Dong, P. Guzdar, and C. Liu, Phys. Fluids 30, 1331 (1987).

[13] A. Dimits et al., Phys. Plasmas 7, 969 (2000).

[14] F. Jenko, W. Dorland, M. Kotschenreuther, and B. Rogers, Phys. Plasma 7, 1904 (2000).

[15] E. Belli, Ph.D. dissertation, Princeton University (2006).

[16] P. Catto, W. Tang, and D. Baldwin, Plasma Phys. 23, 639 (1981).

[17] P. Catto and K. Tsang, Phys. Fluids 20, 396 (1977).

[18] R. Miller, M. Chu, J. Greene, Y. Lin-Liu, and R. Waltz., Phys. Plasmas 5, 973 (1998).

[19] S. Zheng, A. Wootton, and E. Solano, Phys. Plasmas 3, 1176 (1996).

[20] G. McKee et al., Phys. Plasmas 10, 1712 (2003).

[21] T. Rhodes et al., Phys. Plasmas 9, 2141 (2002).

[22] R. Waltz, Private Communication (2006). 